\title{
High Level Process Modelling: A Key Feature of Any BPR Project
}

\author{
A. W. Chan* P. Bradley** \\ * National Research Council (NRC), Ottawa,Ontario, Canada, \\ Tel: +1-613-993-9437 E-mail:A_Chan@syslab.nrc.ca \\ **CIMRU, University College Galway, Ireland. \\ Tel: +353-91-750414 E-mail:Padraig.Bradley@ucg.ie
}

\begin{abstract}
A wide range of software tools are available for use in BPR programmes. This paper debates some of the issues involved in using enterprise modelling tools. Additionally, a specific enterprise modelling tool, and its use in two case studies, is described.
\end{abstract}

\section{Keywords}

Business process reengineering, enterprise modelling, EMS, BPR

\section{INTRODUCTION}

Business Process Reengineering (BPR) is an approach for achieving dramatic improvements in a company's performance in a relatively short period of time. BPR has helped companies to understand how the different functions or processes in their business are related. Many approaches exist for improving performance in manufacturing (JIT, OPT, etc.) but few approaches offer the opportunity to make dramatic improvements in the non-manufacturing or "white-collar" areas of a company.

Some of the goals of BPR include (Classe, 1994):

- achieving step changes in performance,

- moving from a function to a process based capability,

- emphasising customer focus,

- integrating work,

- developing a process management culture.

High level process modelling is a very helpful, perhaps necessary, first step in any Business Process Reengineering (BPR) project. It provides a global view of how key 
processes in an enterprise are interrelated, and predicts the potential impact of reengineering lower level processes. Also, it provides a framework for incorporating new process modules and facilitates in the migration from 'As Is' to 'To Be' scenarios as processes are reengineered in an organisation.

\section{BUSINESS PROCESS REENGINEERING}

Reengineering was a term coined by Michael Hammer in 1990 to describe the process of change that certain organisations were undertaking in order to achieve dramatic performance improvements. The improvements being sought were dramatic and radical in nature and these companies were able to attain significant performance improvements while also totally changing the focus of the organisation. Hammer himself says, "... I never say that I invented reengineering. What I say is that I may have discovered it, which is an entirely different thing" (Barrier, 1994).

Hammer \& Champy define reengineering as "the fundamental rethinking and radical redesign of business processes to achieve dramatic improvements in critical, contemporary measures of performance, such as cost, quality, service and speed" (Hammer \& Champy, 1993). The key words in this definition, which form the basis of the reengineering approach are:

- fundamental,

- radical,

- dramatic,

- business processes.

Reengineering begins with no assumptions, and the most fundamental (or basic) questions about the companies, and how they operate, need to be asked. Reengineering programs should concentrate on what the company should be doing and should ignore what the company is currently doing. Reengineering is about redesigning the way that the company does business. This involves becoming process oriented. In reengineering, radical redesign means disregarding existing structures and procedures and inventing completely new ways of accomplishing work. This is done by starting with a 'clean slate' and redesigning the processes to reflect these new approaches. Hammer argues for the 'neutron bomb' approach of obliteration and argues strongly against automating the current ways of doing business (Hammer, 1990).

Reengineering is also about achieving dramatic performance improvements (Hammer \& Champy, 1993). The levels of improvement sought need to be multiplicative and not fractional (10X rather than 10\%) (Davenport, 1993). The dramatic improvements required means that reengineering must be undertaken as an all or nothing proposition. The processes should be redesigned in such a way as to allow these dramatic improvements to be achieved.

Reengineering must focus on redesigning fundamental business processes and not on departments or other organisational units. Most businesses are not 'process oriented'; they are focused on tasks, on jobs, on people and on structures, but not on processes. A business process can be viewed as a set of logically related tasks performed to achieve a 
definite business outcome (Davenport \& Short, 1990). These business processes have two important characteristics:

1. they have customers, and:

2. they cross organisational boundaries.

As one of the primary concerns of reengineering are business processes, reengineering is often known as business process reengineering, or simply BPR.

Customer order fulfilment is a good example of a business process as the output of the process (i.e. product/service) is required by a customer and the process itself spans several departments in the organisation. BPR involves reengineering these processes to achieve radical improvements in cost, time, responsiveness, performance, quality, etc. These reengineered processes should provide the company with dramatic improvements in cost, response time, and performance, as well as reflecting the company's strategy. The focus in BPR is on 'why' a particular process activity is undertaken.

Processes, not organisations, are the object of re-engineering. All work is performed as part of a process, but often these processes tend to be invisible, fragmented and unmanaged. This is due to the fact that these processes are spread across different organisations with many people working on small pieces of the overall process. No one person manages the overall process and all personnel working on a process identify themselves as working in a particular department but not on a particular process.

\section{HIGH LEVEL PROCESS MODELLING}

The general requirement that simulation models be specified and validated at a very basic level of detail has been a source of frustration in many modelling exercises. It is often desirable to develop 'quick and dirty' models to obtain ballpark results and to do sensitivity analysis without undertaking a detailed study leading to detailed specifications of the processes in the system to be modelled. Alternative actions can be evaluated at a coarse level, and a small set of selected actions may then be investigated in greater detail.

High level process modelling implies modelling the key processes in an enterprise, including business and manufacturing and/or service processes, by taking a holistic view of these processes to include their interactions with one another and their overall impact on key measures of effectiveness for the enterprise. Hierarchical decomposition, which allows the specification of processes and activities in successively greater detail, is a useful concept in process modelling, and high level process modelling is a useful first step in this procedure. In enterprise modelling studies, the objectives may be to assess the overall performance of the system, to examine the balance between work groups, to study material supply and logistics issues, or to look at overall plant decision making, such as planning and scheduling procedures. The interactions with the outside world and various measures of effectiveness may have to be considered. High level process modelling provides the basis for some activities carried out within the system to be modelled in great detail while others are left in broad terms.

Whereas lower level modelling is often concerned with material flows, high level process modelling is more likely to emphasise the modelling of information flows. High level 
process modelling should be planned so as to allow progressive refinement of the model over time. This implies assuring that the consistency in the interactions between different segments in the model can be maintained. Boundaries and interfaces must be carefully defined so that one part of the model can be modified without invalidating other parts of the model. In defining the boundary of an object, the consistency of information should be a concern. For example, a department modelled at a detailed level may require data from the same department modelled at an abstract level, or vice versa.

High level process modelling can be part of a top-down or bottom-up approach to process modelling. In the former case, it is part of a process of improved definition of a model. Until a process, activity or function is decomposed into elements whose behaviour can be defined in detail, there will be some uncertainty about the accuracy with which it should be represented in the model. Sensitivity analysis may reveal which parts must be broken down into greater detail to ensure reliability of the results. In the latter case, subsystems which have been modelled in detail and already validated could be aggregated to form a larger model. For example, cell models might be incorporated into a shop floor model; shop floor models could be combined to form a plant model, etc. The details within the sub-model might be hidden in the higher level model by aggregating the summary statistics generated while running the detailed models.

High level process modelling may also involve approximation by treating activities in less detail than in real life. For example, an activity such as Process Workpiece on Machine $X$ consists of at least three sub-activities, loading the workpiece into the machining position, machining it, and unloading it from the machining position. Where detailed statistics on resource utilisation are not being sought, the simplification is adequate for modelling the start and finish of processing the workpiece.

Reengineering (or BPR) must begin with a survey of the process landscape to identify processes that are candidates for reengineering (Davenport, 1993). The key activities in identifying a process for re-engineering are as follows:

- Enumerate major processes,

- Determine process boundaries,

- Assess strategic relevance of each process,

- Render high-level judgements of the "health" of each process,

- Qualify the culture and politics of each process.

When the objective is radical process change, a process should be defined as broadly as possible (Davenport, 1993). The fewer and broader the processes, the greater the scope for reengineering success, while the problems of understanding, measuring and changing the process are also greater. Once the processes have been identified at a high level, the boundaries between these processes need to be managed. The following questions may help in defining these boundaries:

- When should the process owner's concern with the process begin and end?

- When should process customers' involvement begin and end? 
- Where do processes begin and end?

- Is the process fully embedded within another process?

- Are performance benefits likely to result from combining the process with other processes or subprocesses?

What processes should be chosen for re-engineering? Processes that may be suited for re-engineering can be grouped into three types (Hammer \& Champy, 1993), namely:

1. Broken processes,

2. Important processes,

3. Feasible processes.

Broken processes are generally well known and have some common symptoms, which include:

- extensive information exchange, data redundancy and rekeying,

- inventory, buffers and other assets,

- high ratio of checking and control to value adding,

- rework and iteration,

- complexity, exceptions and special cases.

Important processes tend to impact on outside customers. These processes contain points where the customer (on whom the organisation depends) has contact with the organisation. These processes should be customer oriented and capable of delivering the performance required by the customer. Feasible processes are determined by sets of factors that determine the likelihood of success of a particular re-engineering effort. One such factor is cost, as high cost reduces feasibility. Once a process has been selected, it should be properly understood through careful analysis.

Hammer has identified seven principles that should guide any business process reengineering exercise undertaken (Hammer, 1990). These principles, shown below, can be used as a guide in developing high quality 'To-be' process configurations.

1. Organise about outcomes, not tasks.

2. Have those who use the output of the process perform the process.

3. Subsume information processing work into the real work that produces the information.

4. Treat geographically dispersed resources as though they were centralised.

5. Link parallel activities instead of integrating their results.

6. Put the decision point where the work is performed, and build control into the process.

7. Capture information once and at the source.

In any BPR project, it is important to gain an overall understanding of how the key processes in an enterprise relate to one another before focusing in on one or a few processes to reengineer. This is important both for the 'obliterate' approach or the 'migrate' approach commonly used in reengineering exercises. In the former case, the high level process modelling will provide a 'birdseye' view of the philosophy and culture of the company, underscoring any obvious areas where new technology, such as information technology, can play a significant role in reengineering. In the latter case, the high level, 
enterprise-wide process model will help to highlight the key decision points and bottlenecks in the system, thereby pinpointing processes and process steps which should receive the most attention. In both cases, the value-add of each process step will be demarcated. Not only can non-value-adding steps be identified for elimination, but it can also be predicted where the most impact can be expected when value-adding steps are reengineered.

Many companies have started to look beyond the four walls of their organisation, and are attempting to integrate their customers and suppliers into their value chains and systems. The interactions between the processes in an enterprise and external agencies, such as customers and suppliers, can be much more appropriately captured with high level process modelling. With the insight gained from these models, BPR can be carried out in such a way that customer viewpoints and supplier system capabilities will be explicitly considered rather than as an afterthought.

\section{USE OF A PROCESS MODELLING TOOL}

The development of a high level model of the business process of an organisation can help in understanding how the processes relate to each other. The activities in the process, the process boundaries, the resources used in the process all need to be understood. Due to the amount of detail, and the number of iterations involved in developing a reasonably detailed high level model, the use of a modelling tool offers specific benefits. A properly chosen process modelling tool satisfies the following criteria (Davenport, 1993):

1. It is fast and easy to use at a high level;

2. It is applicable to the portrayal and analysis of the new process, enabling new and old processes to be compared in the same formats and perhaps even driven by the same set of simulation variables;

3. It provides not only a descriptive, but also an analytical, model of the process, facilitating an understanding of such factors as time, cost, and other resources consumed by the process; and

4. It supports the addition of successive levels of systems and data-oriented detail, enabling it ultimately (and seamlessly) to serve a useful purpose during the systems design and/or prototyping stages.

An integrated modelling tool that will permit modelling the processes in an organisation at various levels with flexibility is thus very desirable. Ideally, the same tool can be used to model processes at a very high level, giving a global view of how processes relate to one another, as well as to model low level processes in great detail, allowing these processes to be finetuned for maximum operational efficiency.

The Enterprise Modelling System (EMS), jointly developed by the National Research Council of Canada and a consortium of companies, is an example of a modelling tool available to build multi-level models of processes found in an enterprise (Chan, 1994). It can facilitate the establishment of a business or manufacturing strategy for an enterprise. This should be a dynamic process which allows periodic updates to the strategy by taking into account the changing conditions of external variables, such as competition, consumer 
taste, economic environment, and unpredictable occurrences in the marketplace. The EMS provides special features to model processes from an overall enterprise-wide level, to processes distributed in multiple plants at different geographical locations, to processes spanning different departments in a plant, to manufacturing processes found in various manufacturing cells in a department, and all the way to control processes for manufacturing and material handling equipment within a manufacturing cell. Not only can process models be created at the various levels in the enterprise hierarchy, but a combination of process models of varying degrees of details can be set up within the same framework, and made to run together by passing the output of one model as input to another model. This is particularly useful when the user would like to home in on the details of one process, while maintaining a high level view of the overall picture.

The EMS permits a range of levels of abstraction in model building by providing high level constructs (templates and modules) rather than restricting the user to basic block commands. A single organisation unit may represent a plant or warehouse in a high level model, and may be exploded into a whole set of resources and activities at a lower level. Common measures of performance which are tracked by the EMS are time, cost and value. A breakdown of the time, cost and value for each subprocess or activity in the definition of a high level process is available to the user. Whereas time is based on the estimated duration of an activity specified by the user, cost is computed based on a fixed cost and a variable cost, which in turn is derived from the unit cost of a resource. Value is assigned by the user to reflect the value an activity is deemed to be worth to the customer, providing a basis to examine the value added by each component in a value chain. In all cases, either fixed values, values within a range, or values based on statistical distributions can be used. These measures of performance provide the user with a high level view of the expected impact of process changes, facilitating decisions to focus in on specific subprocesses or activities.

\section{TWO EXAMPLES}

The Enterprise Modelling System has been used in the case study of a multi-plant manufacturer for the high level process modelling of the order fulfilment process. Key processes corresponding to functions in marketing, operations, receiving, shipping, production and stores have been modelled at a coarse level. This has provided not only a useful documentation for the current processes in the organisation, but also a basis for Business Process Reengineering studies in the future, and for selecting processes for indepth review.

An example of a high level process in the Order Fulfilment process definition is the Fill Materials Order process. This process is broadly broken down into five subprocesses as shown in Figure 1. The Receive Materials Request subprocess has the resource Stores Supervisor receiving a Materials Request from either the production shop floor or from another plant in the enterprise. The next subprocess, Generate Move Documentation, has the Stores Supervisor taking the input Materials Request and transforming it into the output, Materials Move Documentation. The next subprocess, Retrieve Materials, involves taking the Materials Move Documentation to the Storeman and deciding whether 


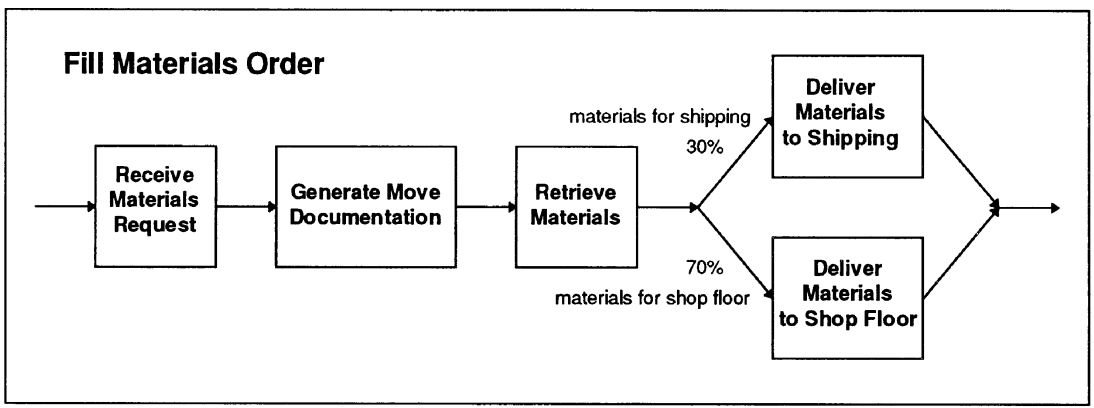

Figure 1 Example of a High Level Process.

the material requested is for the shop floor or for another plant. Depending on the nature of the request, the Storeman transfers the materials requested to the proper destination, either the shipping department or the production shop floor. Rather than modelling the attributes of each Materials Request in detail to determine which branch in the process definition should be taken, the high level process model simply assigns a probability for each of the ensuing branches (see Figure 1). Subsequent refinement of the model may warrant modelling the branching decision in greater detail.

While resources employed in the execution of the Fill Materials Order process, such as Stores Supervisor, Storeman, Shipping Staff, etc. have been specified with their respective position in the organisation structure, unit cost, capabilities, etc., the high level process model is not designed to track the utilisation of any individual resource. In other words, these resources are treated in an aggregate manner, with one designation representing possibly a whole group of similar resources, and their individual identities are not explicitly modelled. With the help of overall summary information such as time and cost charts (Figure 2), the user will get a high level view of the relative impact of changes made to each subprocess. This will form the basis of re-engineering alternatives, as well as decisions to go to lower levels of detail for selected subprocesses, such as the Retrieve Materials subprocess. In such a case, the user would be concerned with the actual retrieval of the requested materials, and the logistics of transporting to the proper location, possibly including the use of materials handling devices, and so on.

FirstSTEP, a commercially available business process re-engineering tool based on the EMS technology, has been adopted by a major financial institution to model high level processes in the Global Custody Services it offers to its clients in both mature and emerging markets throughout the world. Key components of these services include asset safekeeping, trade settlement, income collection, corporate action processing and accounting. High levels of customisation and exception processing are involved, which in turn lead to high levels of customer contact and inquiry processing, requiring the definition of complex support processes. 


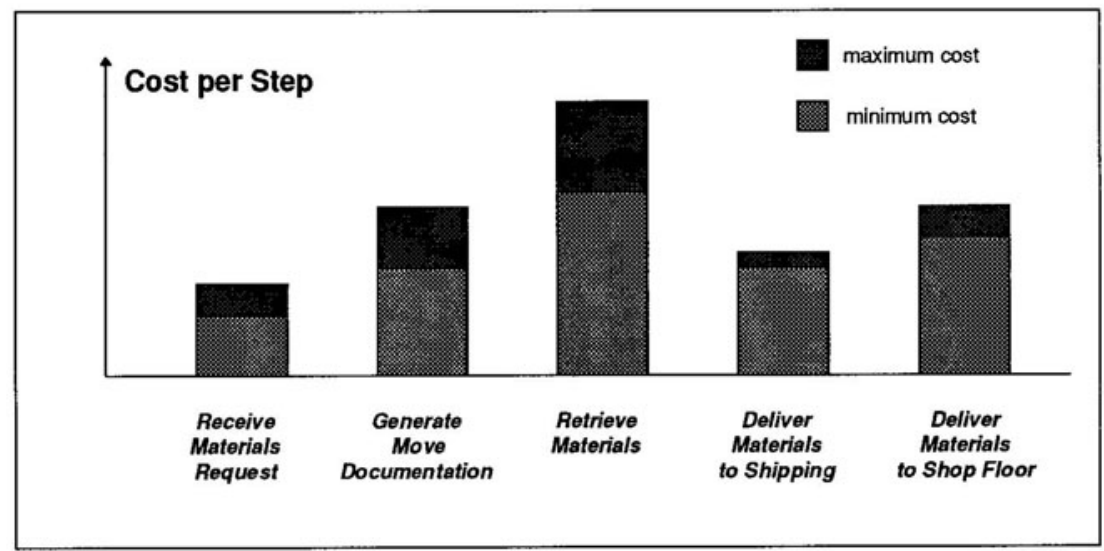

Figure 2 Example of a Process Cost Chart.

A high level model of the current processes is being constructed using FirstSTEP, facilitating the forecast of capacity requirements and the identification of process bottlenecks and streamlining opportunities. Impact analysis of re-engineered processes and possible enhancements to the system are being carried out within the safety of a 'virtual' environment. Other areas of investigation planned using the model include bottlenecks caused by redundant controls, backlogs resulting from rigid work distribution rules, and the elimination of 'black holes' or unnecessary hand-offs. It is expected that the high level process model will provide a valuable resource for future re-engineering and capacity modelling activities within the Customer Service Unit in the company.

\section{Conclusion}

In general, modelling the processes found in an enterprise at a high level will provide useful insight into the relationships between the key processes. As well, it will help to highlight the bottlenecks so that they can be the focus of attention in process redesign. The cost effectiveness of reengineering certain processes can also be assessed, based on the relative cost and time impact of various processes on the organisation. The high level process model developed can provide a framework whereby modules of individual processes with much greater detail can be plugged in, and the overall impact on the company can be studied. The use of a flexible and integrated process modelling tool will greatly facilitate this exercise. 


\section{$8 \quad$ References}

Barrier, M.(1994), Re-engineering Your Company, Nation's Business, pp: 1622.

Chan, A.W.(1994), Enterprise Modelling, Modern Manufacturing: Information Control and Technology, (ed. M. Zaremba, B. Prasad), Springer-Verlag, London.

Classe, A. (1994), Software Tools for Re-engineering, Business Intelligence, London.

Davenport, T. H. (1993). Process Innovation: Reengineering Work through Information Technology, Harvard Business School Press, Boston.

Davenport, T. H., Short, J. E. (1990), The New Industrial Engineering:

Information Technology and Business Process Redesign, Sloan Management Review, pp: 11-27.

Hammer, M. (1993), Reengineering Work: Don't Automate, Obliterate, Harvard Business Review, pp: 104-112, Boston.

Hammer, M., Champy, J.(1993), Reengineering the Corporation: A Manifesto for Business Revolution, Harper Business Press, New York..

\section{Biography}

Albert W. Chan is a Senior Research Officer in the Institute for Advanced Manufacturing Technology at the National Research Council of Canada. He received a Bachelor of Science in Mathematics from the University of Minnesota, and a MSc and $\mathrm{PhD}$ in Industrial Engineering from the University of Florida. His research interests include simulation and computer modelling, decision support systems, and process modelling and re-engineering of business and industrial systems.

Padraig Bradley is a Senior Research Engineer in the Computer Integrated Manufacturing Research Unit (CIMRU) at University College Galway. He has obtained a Bachelor and Masters degree in Engineering from University College Galway. His research interests lie in the areas of manufacturing systems, knowledge based systems and business process reengineering. 